\title{
A Compact Difference Scheme for Solving Fractional Neutral Parabolic Differential Equation with Proportional Delay
}

\author{
Wei Gu, ${ }^{1}$ Yanli Zhou, ${ }^{2}$ and Xiangyu Ge ${ }^{1}$ \\ ${ }^{1}$ School of Statistics \& Mathematics, Zhongnan University of Economics and Law, Wuhan 430073, China \\ ${ }^{2}$ School of Finance, Zhongnan University of Economics and Law, Wuhan 430073, China \\ Correspondence should be addressed to Yanli Zhou; ylzhou8507@gmail.com and Xiangyu Ge; xiangyu_ge@163.com
}

Received 5 July 2017; Accepted 17 September 2017; Published 18 October 2017

Academic Editor: Xinguang Zhang

Copyright (c) 2017 Wei Gu et al. This is an open access article distributed under the Creative Commons Attribution License, which permits unrestricted use, distribution, and reproduction in any medium, provided the original work is properly cited.

\begin{abstract}
A linearized compact finite difference scheme is constructed for solving the fractional neutral parabolic differential equation with proportional delay. By the energy method, the unconditional stability of the scheme is proved, and the convergence order of the scheme is proved to be $O\left(\tau^{2-\alpha}+h^{4}\right)$. A numerical test is also conducted to validate the accuracy and efficiency of the numerical algorithm.
\end{abstract}

\section{Introduction}

In the past few years, more and more scholars have been attracted to the research of delay partial differential equations (DPDEs) [1-3]. However, most DPDEs have no exact solutions. Constructing efficient numerical methods for DPDEs is of great importance [4-7]. For details on numerically solving neutral delay parabolic differential equations (NDPDEs), the reader is referred to $[5,8]$. Recently, fractional delay partial differential equations have been of great interest due to their application in automatic control, population dynamics, economics, and so forth $[9,10]$. For details on numerical solutions to fractional delay partial differential equations, we refer the reader to $[11,12]$. The work in [11] considers the numerical method without theoretical analysis, and the work in [12] considers the numerical method for a type of semilinear fractional partial differential equation with time delay.

In this paper, we consider the following fractional neutral parabolic differential equation with proportional delay:

$$
\begin{aligned}
\frac{\partial^{\alpha} w}{\partial t^{\alpha}}= & D\left(\frac{\partial^{2} w(x, t)}{\partial x^{2}}+\frac{\partial^{2} w(x, p t)}{\partial x^{2}}\right) \\
& +f_{w}(w(x, t), w(x, p t), x, t), \\
& (x, t) \in(0,1) \times[0, T],
\end{aligned}
$$

$$
\begin{aligned}
& w(0, t)=\gamma_{w}(t), \\
& w(1, t)=\beta_{w}(t),
\end{aligned}
$$$$
t \in(0, T] \text {, }
$$

where $D>0$ is a constant, $0<p<1$.

Let $u(x, t)=w\left(x, e^{t}\right)$ for $t \geq t_{0}+\ln (p)$, where $t_{0} \geq 0$. Then, $u(x, t)$ satisfies the following equation:

$$
\begin{aligned}
& \frac{\partial^{\alpha} u}{\partial t^{\alpha}}= D\left(\frac{\partial^{2} u(x, t)}{\partial x^{2}}+\frac{\partial^{2} u(x, t-s)}{\partial x^{2}}\right) \\
&+f_{w}\left(u(x, t), u(x, t-s), x, e^{t}\right), \\
& \quad(x, t) \in(0,1) \times\left(t_{0}, T\right], \\
& \begin{aligned}
u(x, t)= & \phi_{w}\left(x, e^{t}\right), \quad x \in[0,1], t \in\left[-s, t_{0}\right], \\
u(0, t)= & \gamma_{w}\left(e^{t}\right), \\
u(1, t)= & \beta_{w}\left(e^{t}\right), \\
& \quad t \in\left(t_{0}, T\right],
\end{aligned}
\end{aligned}
$$

where $s=-\ln (p)$. 
For simplicity, we consider the following fractional neutral parabolic differential equation with delay instead of (1):

$$
\begin{aligned}
\frac{\partial^{\alpha} u}{\partial t^{\alpha}}= & D\left(\frac{\partial^{2} u(x, t)}{\partial x^{2}}+\frac{\partial^{2} u(x, t-s)}{\partial x^{2}}\right) \\
& +f(u(x, t), u(x, t-s), x, t), \\
u(x, t)= & \phi(x, t), \quad x \in[0,1], t \in[-s, 0], \\
u(0, t)= & \gamma(t), \\
u(1, t)= & \beta(t),
\end{aligned}
$$

$$
t \in(0, T]
$$

where $s>0$ is a constant delay term. Time fractional partial derivative $\left(\partial^{\alpha} u / \partial t^{\alpha}\right)(0<\alpha<1)$ is defined in the Caputo sense by the following:

$$
\frac{\partial^{\alpha} u}{\partial t^{\alpha}}=\frac{1}{\Gamma(1-\alpha)} \int_{0}^{t}(t-\xi)^{-\alpha} \frac{\partial u(x, \xi)}{\partial \xi} d \xi
$$

where $\Gamma($ ) is the Gamma function.

In this paper, a linearized compact finite difference scheme is constructed for solving (3)-(5). By the energy method, the unconditional stability of the scheme is then proved, and the convergence order of the scheme is proved to be $O\left(\tau^{2-\alpha}+h^{4}\right)$. A numerical test is also conducted to validate the accuracy and efficiency of the numerical algorithm.

The rest of the paper is organized as follows. In Section 2, a compact difference scheme is constructed to solve (3)-(5). Section 3 considers the solvability, convergence, and stability of the provided difference scheme. In Section 4, a numerical test is presented to illustrate the validity of the theoretical results. Section 5 gives a brief conclusion of this paper.

\section{The Construction of the Compact Difference Scheme}

Throughout this paper, assume $u(x, t) \in C^{6,2}([0,1] \times(0, T])$. Function $f(u(x, t), u(x, t-s), x, t)$ is sufficiently smooth and satisfies

$$
\begin{aligned}
& \left|f\left(\mu+\epsilon_{1}, \nu+\epsilon_{2}, x, t\right)-f(\mu, \nu, x, t)\right| \\
& \quad \leq c_{0}\left|\epsilon_{1}\right|+c_{1}\left|\epsilon_{2}\right|,
\end{aligned}
$$

where $\epsilon_{1}, \epsilon_{2}$ are arbitrary real numbers and $c_{0}$ and $c_{1}$ are positive constants.

First, let $M$ and $N$ be two positive integers; then, we take $h=1 / M, \tau=s / n$ ( $n>0$ is a positive integer), $x_{i}=i h$, $t_{k}=k \tau$. Define $\Omega_{h \tau}=\Omega_{h} \times \Omega_{\tau}$, where $\Omega_{h}=\left\{x_{i} \mid 0 \leq\right.$ $i \leq M\}, \Omega_{\tau}=\left\{t_{k} \mid-n \leq k \leq N\right\}, N=[T / \tau]$. Denote $U_{i}^{k}=u\left(x_{i}, t_{k}\right), 0 \leq i \leq M,-n \leq k \leq N$ throughout this paper. Let

$$
\mathscr{W}=\left\{v_{i}^{k} \mid 0 \leq i \leq M,-n \leq k \leq N\right\}
$$

be the grid function space defined on $\Omega_{h \tau}$. The following notations are used:

$$
\begin{aligned}
& \delta_{x} v_{i+1 / 2}^{k}=\frac{v_{i+1}^{k}-v_{i}^{k}}{h}, \\
& \delta_{x}^{2} v_{i}^{k}=\frac{v_{i+1}^{k}-2 v_{i}^{k}+v_{i-1}^{k}}{h^{2}} \\
& \mathscr{A} v_{i}^{k}=\frac{1}{12}\left(v_{i-1}^{k}+10 v_{i}^{k}+v_{i+1}^{k}\right), \\
& \delta_{t}^{\alpha} v^{k}=\frac{\tau^{-\alpha}}{\Gamma(2-\alpha)}\left[d_{0} v^{k}-\sum_{j=1}^{k-1}\left(d_{k-j-1}-d_{k-j}\right) v^{j}\right.
\end{aligned}
$$

$$
\left.-d_{k-1} v^{0}\right]
$$

where $d_{j}=(j+1)^{1-\alpha}-j^{1-\alpha}, j \geq 0$.

For the time fractional derivative, we have the following lemma.

Lemma 1 (see [13]). Suppose $0<\alpha<1, y \in C^{2}\left[0, t_{k}\right]$; it holds that

$$
\begin{aligned}
& \mid \frac{1}{\Gamma(1-\alpha)} \int_{0}^{t_{k}} \frac{y^{\prime}(s) d s}{\left(t_{k}-s\right)^{\alpha}}-\frac{\tau^{-\alpha}}{\Gamma(2-\alpha)}\left[d_{0} y\left(t_{k}\right)\right. \\
& \left.\quad-\sum_{j=1}^{k-1}\left(d_{k-j-1}-d_{k-j}\right) y\left(t_{j}\right)-d_{k-1} y\left(t_{0}\right)\right] \mid \\
& \leq \frac{1}{\Gamma(2-\alpha)}\left[\frac{1-\alpha}{12}+\frac{2^{2-\alpha}}{2-\alpha}-\left(1+2^{-\alpha}\right)\right] \\
& \quad \cdot \max _{0 \leq t \leq t_{k}}\left|y^{\prime \prime}(t)\right| \tau^{2-\alpha},
\end{aligned}
$$

and $d_{j}$ satisfies the following lemma.

Lemma 2 (see [14]). Assume $0<\alpha<1$; then, it holds that

(1) $d_{j}$ decreases monotonically as $j$ increases, and $0<d_{j} \leq$ 1 ;

(2) $d_{0}=1, \sum_{j=1}^{k-1}\left(d_{k-j-1}-d_{k-j}\right)=d_{0}-d_{k-1}$.

Lemma 3 (see [5]). Suppose $y(x) \in C^{6}\left[x_{i-1}, x_{i+1}\right]$; then, one has

$$
\begin{aligned}
\frac{1}{12} & {\left[y^{\prime \prime}\left(x_{i-1}\right)+10 y^{\prime \prime}\left(x_{i}\right)+y^{\prime \prime}\left(x_{i+1}\right)\right] } \\
& -\frac{1}{h^{2}}\left[y\left(x_{i-1}\right)-2 y\left(x_{i}\right)+y\left(x_{i+1}\right)\right] \\
= & \frac{h^{4}}{240} y^{(6)}\left(\iota_{i}\right),
\end{aligned}
$$

where $\iota_{i} \in\left(x_{i-1}, x_{i+1}\right)$. 
Considering (3) at the point $\left(x_{i}, t_{k}\right)$, we have

$$
\begin{aligned}
& \frac{\partial^{\alpha} u}{\partial t^{\alpha}}\left(x_{i}, t_{k}\right)= D\left(\frac{\partial^{2} u}{\partial x^{2}}\left(x_{i}, t_{k}\right)+\frac{\partial^{2} u}{\partial x^{2}}\left(x_{i}, t_{k}-s\right)\right) \\
&+f\left(u\left(x_{i}, t_{k}\right), u\left(x_{i}, t_{k}-s\right), x_{i}, t_{k}\right), \\
& 0 \leq i \leq M, 0 \leq k \leq N .
\end{aligned}
$$

From Lemma 1, we obtain

$$
\frac{\partial^{\alpha} u}{\partial t^{\alpha}}\left(x_{i}, t_{k}\right)=\delta_{t}^{\alpha} U_{i}^{k}+r_{i}^{k}
$$

where

$$
\begin{aligned}
r_{i}^{k}= & \frac{1}{\Gamma(2-\alpha)}\left[\frac{1-\alpha}{12}+\frac{2^{2-\alpha}}{2-\alpha}-\left(1+2^{-\alpha}\right)\right] \\
& \cdot \max _{0 \leq t \leq t_{k}}\left|\frac{\partial^{2} u\left(x_{i}, t_{k}\right)}{\partial t^{2}}\right| \tau^{2-\alpha} .
\end{aligned}
$$

From Taylor expansion, we have

$$
\begin{aligned}
& f\left(u\left(x_{i}, t_{k}\right), u\left(x_{i}, t_{k}-s\right), x_{i}, t_{k}\right) \\
& =f\left(2 U_{i}^{k-1}-U_{i}^{k-2}, U_{i}^{k-n}, x_{i}, t_{k}\right) \\
& \quad+\tau^{2} \frac{\partial^{2} u}{\partial t^{2}}\left(x_{i}, \eta^{k}\right) f_{\mu}\left(\varrho_{i}^{k}, U_{i}^{k-n}, x_{i}, t_{k}\right),
\end{aligned}
$$

where $\eta^{k} \in\left(t_{k-2}, t_{k}\right), \varrho_{i}^{k}$ in between $u\left(x_{i}, t_{k}\right)$ and $2 U_{i}^{k-1}-U_{i}^{k-2}$.

Substituting (13) and (15) into (12) and applying the operator $\mathscr{A}$ on both sides of (12), we obtain

$$
\begin{aligned}
\mathscr{A} \delta_{t}^{\alpha} U_{i}^{k}= & D\left(\mathscr{A} \frac{\partial^{2} u}{\partial x^{2}}\left(x_{i}, t_{k}\right)+\mathscr{A} \frac{\partial^{2} u}{\partial x^{2}}\left(x_{i}, t_{k}-s\right)\right) \\
& +\mathscr{A} f\left(2 U_{i}^{k-1}-U_{i}^{k-2}, U_{i}^{k-n}, x_{i}, t_{k}\right)+\mathscr{A} R_{0 i}^{k},
\end{aligned}
$$

where

$$
R_{0 i}^{k}=r_{i}^{k}+\tau^{2} \frac{\partial^{2} u}{\partial t^{2}}\left(x_{i}, \eta^{k}\right) f_{\mu}\left(\varrho_{i}^{k}, U_{i}^{k-n}, x_{i}, t_{k}\right) .
$$

From Lemma 3 and Taylor expansion, we have

$$
\begin{aligned}
& \mathscr{A} \frac{\partial^{2} u}{\partial x^{2}}\left(x_{i}, t_{k}\right)=\delta_{x}^{2} U_{i}^{k}+\frac{h^{4}}{240} \frac{\partial^{6} u}{\partial x^{6}}\left(\theta_{i}^{k}, t_{k}\right), \\
& \theta_{i}^{k} \in\left(x_{i-1}, x_{i+1}\right), \\
& \mathscr{A} \frac{\partial^{2} u}{\partial x^{2}}\left(x_{i}, t_{k}-s\right)=\delta_{x}^{2} U_{i}^{k-n}+\frac{h^{4}}{240} \frac{\partial^{6} u}{\partial x^{6}}\left(\theta_{i}^{k-n}, t_{k-n}\right), \\
& \theta_{i}^{k-n} \in\left(x_{i-1}, x_{i+1}\right) .
\end{aligned}
$$

Substituting (18) into (16), we have

$$
\begin{aligned}
\mathscr{A} \delta_{t}^{\alpha} U_{i}^{k}= & D\left(\delta_{x}^{2} U_{i}^{k}+\delta_{x}^{2} U_{i}^{k-n}\right) \\
& +\mathscr{A} f\left(2 U_{i}^{k-1}-U_{i}^{k-2}, U_{i}^{k-n}, x_{i}, t_{k}\right)+R_{i}^{k},
\end{aligned}
$$

where

$$
\begin{aligned}
R_{i}^{k}= & \mathscr{A} R_{0 i}^{k}+\frac{h^{4}}{240} \frac{\partial^{6} u}{\partial x^{6}}\left(\theta_{i}^{k}, t_{k}\right) \\
& +\frac{h^{4}}{240} \frac{\partial^{6} u}{\partial x^{6}}\left(\theta_{i}^{k-n}, t_{k-n}\right) .
\end{aligned}
$$

Noticing $u(x, t) \in C^{6,2}([0,1] \times(0, T])$ and $(7)$, we can easily obtain

$$
\left|R_{i}^{k}\right| \leq C_{R}\left(\tau^{2-\alpha}+h^{4}\right), \quad 0 \leq i \leq M, \quad 1 \leq k \leq N .
$$

Discretizing the initial and boundary conditions of (4) and (5), we obtain

$$
\begin{aligned}
U_{i}^{k} & =\phi\left(x_{i}, t_{k}\right), \quad 0 \leq i \leq M,-n \leq k \leq 0, \\
U_{0}^{k} & =\gamma\left(t_{k}\right), \\
U_{M}^{k} & =\beta\left(t_{k}\right),
\end{aligned}
$$

$$
1 \leq k \leq N
$$

Replacing $U_{i}^{k}$ by $u_{i}^{k}$ in (19), (22), and (23) and omitting $R_{i}^{k}$, we can obtain the following compact difference scheme:

$$
\begin{aligned}
\mathscr{A} \delta_{t}^{\alpha} u_{i}^{k}= & D\left(\delta_{x}^{2} u_{i}^{k}+\delta_{x}^{2} u_{i}^{k-n}\right) \\
& +\mathscr{A} f\left(2 u_{i}^{k-1}-u_{i}^{k-2}, u_{i}^{k-n}, x_{i}, t_{k}\right), \\
u_{i}^{k}= & \phi\left(x_{i}, t_{k}\right), \quad 0 \leq i \leq M,-n \leq k \leq 0, \\
u_{0}^{k}= & \gamma\left(t_{k}\right), \\
u_{M}^{k}= & \beta\left(t_{k}\right),
\end{aligned}
$$

$$
1 \leq k \leq N
$$

\section{The Solvability, Convergence, and Stability of the Difference Scheme}

Define the following grid function space on $\Omega_{h}$ :

$$
\mathscr{V}_{h, 0}=\left\{u \mid u=\left(u_{0}, u_{1}, \ldots, u_{M}\right), u_{0}=u_{M}=0\right\} .
$$

If $u, v \in \mathscr{V}_{h, 0}$, we introduce the following inner products and corresponding norms:

$$
\begin{gathered}
(u, v)=h \sum_{i=1}^{M-1} u_{i} v_{i}, \\
\|u\|=\sqrt{(u, u)},\|u\|_{\infty}=\max _{1 \leq i \leq M-1}\left|u_{i}\right|, \\
\left\langle\delta_{x} u, \delta_{x} v\right\rangle=h \sum_{i=0}^{M-1}\left(\delta_{x} u_{i+1 / 2}\right)\left(\delta_{x} v_{i+1 / 2}\right),
\end{gathered}
$$

$$
\left|\delta_{x} u\right|_{1}=\sqrt{\left\langle\delta_{x} u, \delta_{x} u\right\rangle} .
$$

It is easy to obtain the following lemma.

Lemma 4 (see [12]). $\forall u \in \mathscr{V}_{h, 0}$, one has $\|\mathscr{A} u\|^{2} \leq\|u\|^{2}$. 
Lemma 5 (see [15]). $\forall u \in \mathscr{V}_{h, 0}$, one has

$$
\begin{array}{r}
\|u\|_{\infty} \leq \frac{\left|\delta_{x} u\right|_{1}}{2}, \\
\|u\| \leq \frac{\left|\delta_{x} u\right|_{1}}{\sqrt{6}} .
\end{array}
$$

The following lemma will be used in the proof of the stability and convergence analysis.

Lemma 6 (see [15]). Assume that $\left\{F^{k} \mid k \geq 0\right\}$ is a nonnegative sequence and satisfies

$$
F^{k+1} \leq A+B \tau \sum_{i=1}^{k} F^{l}, \quad k=0,1, \ldots
$$

then,

$$
F^{k+1} \leq A \exp (B k \tau), \quad k=0,1,2, \ldots
$$

where $A$ and $B$ are nonnegative constants.

Theorem 7. The difference scheme (24)-(26) has a unique solution.

Proof. Denote $u^{k}=\left[u_{1}^{k}, u_{2}^{k}, \ldots, u_{M-1}^{k}\right]^{T}$; the difference scheme (24)-(26) is a linear tridiagonal system $\mathscr{M} u^{k}=d$, where $d$ only depends on $u^{j}(j \leq k-1), u_{0}^{k}$, and $u_{M}^{k}$ and is independent of $u^{k}$.

$$
\mathscr{M}=\operatorname{tridiag}\left(\frac{1}{12 \lambda}-\frac{D}{h^{2}}, \frac{10}{12 \lambda}+\frac{2 D}{h^{2}}, \frac{1}{12 \lambda}-\frac{D}{h^{2}}\right),
$$

where $\lambda=\tau^{\alpha} \Gamma(2-\alpha)$. We can see that $\mathscr{M}$ is a strictly diagonally dominant coefficient matrix. Thus, scheme (24)-(26) has a unique solution.

To discuss the stability of the difference scheme (24)-(26), we consider the following problem:

$$
\begin{aligned}
\mathscr{A} \frac{\partial^{\alpha} v}{\partial t^{\alpha}}= & D\left(\frac{\partial^{2} v(x, t)}{\partial x^{2}}+\frac{\partial^{2} v(x, t-s)}{\partial x^{2}}\right) \\
& +\mathscr{A} f(v(x, t), v(x, t-s), x, t), \\
& \quad(x, t) \in(0,1) \times(0, T], \\
v(x, t)= & \phi(x, t)+\psi(x, t), \quad x \in[0,1], t \in[-s, 0], \\
v(0, t)= & \gamma(t), \\
u(1, t)= & \beta(t),
\end{aligned}
$$

where $\psi(x, t)$ is the perturbation caused by $\phi(x, t)$. The following difference scheme can be obtained for solving (33):

$$
\begin{aligned}
\mathscr{A} \delta_{t}^{\alpha} v_{i}^{k}= & D\left(\delta_{x}^{2} v_{i}^{k}+\delta_{x}^{2} v_{i}^{k-n}\right) \\
& +\mathscr{A} f\left(2 v_{i}^{k-1}-v_{i}^{k-2}, v_{i}^{k-n}, x_{i}, t_{k}\right), \\
v_{i}^{k}= & \phi\left(x_{i}, t_{k}\right)+\psi_{i}^{k}, \quad 0 \leq i \leq M,-n \leq k \leq 0, \\
v_{0}^{k}= & \gamma\left(t_{k}\right), \\
v_{M}^{k}= & \beta\left(t_{k}\right),
\end{aligned}
$$

$$
1 \leq k \leq N
$$

Denote

$$
\omega_{i}^{k}=v_{i}^{k}-u_{i}^{k}, \quad 0 \leq i \leq M,-n \leq k \leq N .
$$

Definition 8. Assume that $u_{i}^{k}$ satisfies (24)-(26) and $v_{i}^{k}$ satisfies (34)-(36); then, a numerical scheme for (3)-(5) is stable if one has

$$
\left\|\omega^{k}\right\|_{\infty} \leq \bar{C} \max _{-n \leq j \leq 0}\left|\psi^{j}\right|_{1},
$$

where $\bar{C}$ is a bounded constant independent of $h$ and $\tau$.

Theorem 9. Assume $u(x, t) \in C^{6,2}([0,1] \times(-s, T])$ is the solution of (3)-(5); the difference scheme (24)-(26) is stable with respect to the initial perturbation of $\psi_{i}^{k}$; that is, $\left\|\omega^{k}\right\|_{\infty} \leq$ $\widehat{C} \max _{-n \leq j \leq 0}\left|\psi^{j}\right|_{1}$, where $\widehat{C}$ is a positive constant independent of $h$ and $\tau$.

Proof. Subtracting (34)-(36) from (24)-(26), respectively, we can obtain the following equations:

$$
\begin{aligned}
\mathscr{A} \delta_{t}^{\alpha} \omega_{i}^{k} & =D\left(\delta_{x}^{2} \omega_{i}^{k}+\delta_{x}^{2} \omega_{i}^{k-n}\right)+\mathscr{A} q_{i}^{k}, \\
\omega_{i}^{k} & =\psi_{i}^{k}, \quad 0 \leq i \leq M,-n \leq k \leq 0, \\
\omega_{0}^{k} & =0 \\
\omega_{M}^{k} & =0
\end{aligned}
$$

$$
1 \leq k \leq N,
$$

where $q_{i}^{k}=f\left(2 v_{i}^{k-1}-v_{i}^{k-2}, v_{i}^{k-n}, x_{i}, t_{k}\right)-f\left(2 u_{i}^{k-1}-u_{i}^{k-2}\right.$, $\left.u_{i}^{k-n}, x_{i}, t_{k}\right)$.

Multiplying $h \delta_{t}^{\alpha} \omega_{i}^{k}$ on both sides of (39) and summing up for $i$ from 1 to $M-1$, we obtain

$$
\begin{aligned}
h \sum_{i=1}^{M-1}\left(\mathscr{A} \delta_{t}^{\alpha} \omega_{i}^{k}\right)\left(\delta_{t}^{\alpha} \omega_{i}^{k}\right) \\
=D h \sum_{i=1}^{M-1}\left(\delta_{x}^{2} \omega_{i}^{k}+\delta_{x}^{2} \omega_{i}^{k-n}\right)\left(\delta_{t}^{\alpha} \omega_{i}^{k}\right) \\
+h \sum_{i=1}^{M-1}\left(\mathscr{A} q_{i}^{k}\right)\left(\delta_{t}^{\alpha} \omega_{i}^{k}\right),
\end{aligned}
$$


Then, each term of (42) will be estimated. From the discrete Green formula and inequality $-(a+b)^{2} \geq-2\left(a^{2}+b^{2}\right)$, we have

$$
\begin{aligned}
& h \sum_{i=1}^{M-1}\left(\mathscr{A} \delta_{t}^{\alpha} \omega_{i}^{k}\right)\left(\delta_{t}^{\alpha} \omega_{i}^{k}\right) \\
& \quad=\left\|\delta_{t}^{\alpha} \omega^{k}\right\|^{2}+\frac{h^{2}}{12} h \sum_{i=1}^{M-1}\left(\delta_{x}^{2}\left(\delta_{t}^{\alpha} \omega_{i}^{k}\right)\right)\left(\delta_{t}^{\alpha} \omega_{i}^{k}\right) \\
& \quad=\left\|\delta_{t}^{\alpha} \omega^{k}\right\|^{2}-\frac{h^{2}}{12}\left\|\delta_{x} \delta_{t}^{\alpha} \omega^{k}\right\|^{2} \geq \frac{2}{3}\left\|\delta_{t}^{\alpha} \omega^{k}\right\|^{2} .
\end{aligned}
$$

From the discrete Green formula and inequality $-a b \geq-\left(a^{2}+\right.$ $\left.b^{2}\right) / 2$, we have

$$
\begin{aligned}
& D h \sum_{i=1}^{M-1}\left(\delta_{x}^{2} \omega_{i}^{k}\right)\left(\delta_{t}^{\alpha} \omega_{i}^{k}\right) \\
& =-D h \sum_{i=0}^{M-1}\left(\delta_{x} \omega_{i+1 / 2}^{k}\right)\left(\delta_{t}^{\alpha} \delta_{x} \omega_{i+1 / 2}^{k}\right)=-\frac{D}{\lambda} \\
& \cdot h \sum_{i=0}^{M-1}\left(\delta_{x} \omega_{i+1 / 2}^{k}\right)\left[\delta_{x} \omega_{i+1 / 2}^{k}\right. \\
& \left.-\sum_{j=1}^{k-1}\left(d_{k-j-1}-d_{k-j}\right) \delta_{x} \omega_{i+1 / 2}^{j}-d_{k-1} \delta_{x} \omega_{i+1 / 2}^{0}\right] \\
& =-\frac{D}{\lambda}\left\{\left|\delta_{x} \omega^{k}\right|_{1}^{2}\right. \\
& -\sum_{j=1}^{k-1}\left(d_{k-j-1}-d_{k-j}\right)\left\langle\delta_{x} \omega^{k}, \delta_{x} \omega^{j}\right\rangle \\
& \left.-d_{k-1}\left\langle\delta_{x} \omega^{k}, \delta_{x} \omega^{0}\right\rangle\right\} \leq-\frac{D}{\lambda}\left\{\left|\delta_{x} \omega^{k}\right|_{1}^{2}\right. \\
& -\sum_{j=1}^{k-1}\left(d_{k-j-1}-d_{k-j}\right) \frac{\left|\delta_{x} \omega^{k}\right|_{1}^{2}+\left|\delta_{x} \omega^{j}\right|_{1}^{2}}{2} \\
& \left.-d_{k-1} \frac{\left|\delta_{x} \omega^{k}\right|_{1}^{2}+\left|\delta_{x} \omega^{0}\right|_{1}^{2}}{2}\right\}=-\frac{D}{\lambda}\left\{\frac{\left|\delta_{x} \omega^{k}\right|_{1}^{2}}{2}\right. \\
& \left.-\sum_{j=1}^{k-1}\left(d_{k-j-1}-d_{k-j}\right) \frac{\left|\delta_{x} \omega^{j}\right|_{1}^{2}}{2}-d_{k-1} \frac{\left|\delta_{x} \omega^{0}\right|_{1}^{2}}{2}\right\} \text {, } \\
& D h \sum_{i=1}^{M-1}\left(\delta_{x}^{2} \omega_{i}^{k-n}\right)\left(\delta_{t}^{\alpha} \omega_{i}^{k}\right) \\
& =-D h \sum_{i=0}^{M-1}\left(\delta_{x} \omega_{i+1 / 2}^{k-n}\right)\left(\delta_{t}^{\alpha} \delta_{x} \omega_{i+1 / 2}^{k}\right)=-\frac{D}{\lambda}
\end{aligned}
$$

$$
\begin{aligned}
& \cdot h \sum_{i=0}^{M-1}\left(\delta_{x} \omega_{i+1 / 2}^{k-n}\right)\left[\delta_{x} \omega_{i+1 / 2}^{k}\right. \\
& \left.-\sum_{j=1}^{k-1}\left(d_{k-j-1}-d_{k-j}\right) \delta_{x} \omega_{i+1 / 2}^{j}-d_{k-1} \delta_{x} \omega_{i+1 / 2}^{0}\right] \\
& \leq \frac{D}{\lambda}\left\{\frac{1}{4}\left|\delta_{x} \omega^{k}\right|_{1}^{2}+\left|\delta_{x} \omega^{k-n}\right|_{1}^{2}\right. \\
& +\sum_{j=1}^{k-1}\left(d_{k-j-1}-d_{k-j}\right) \frac{\left|\delta_{x} \omega^{k-n}\right|_{1}^{2}+\left|\delta_{x} \omega^{j}\right|_{1}^{2}}{2} \\
& \left.+d_{k-1} \frac{\left|\delta_{x} \omega^{k-n}\right|_{1}^{2}+\left|\delta_{x} \omega^{0}\right|_{1}^{2}}{2}\right\}=\frac{D}{\lambda}\left\{\frac{\left|\delta_{x} \omega^{k}\right|_{1}^{2}}{4}\right. \\
& +\frac{3\left|\delta_{x} \omega^{k-n}\right|_{1}^{2}}{2}+\sum_{j=1}^{k-1}\left(d_{k-j-1}-d_{k-j}\right) \frac{\left|\delta_{x} \omega^{j}\right|_{1}^{2}}{2} \\
& \left.+d_{k-1} \frac{\left|\delta_{x} \omega^{0}\right|_{1}^{2}}{2}\right\} \cdot
\end{aligned}
$$

From the Cauchy-Schwarz inequality, Lemma 4, and (7), we have

$$
\begin{aligned}
h \sum_{i=1}^{M-1}\left(\mathscr{A} q_{i}^{k}\right)\left(\delta_{t}^{\alpha} \omega_{i}^{k}\right) & \\
\leq & \frac{1}{2 \varepsilon}\left\|\delta_{t}^{\alpha} \omega^{k}\right\|^{2} \\
& +\frac{\varepsilon}{2} h \sum_{i=1}^{M-1}\left(\mathscr{A}\left[c_{0}\left|2 \omega_{i}^{k-1}-\omega_{i}^{k-2}\right|+c_{1}\left|\omega_{i}^{k-n}\right|\right]\right)^{2} \\
\leq & \frac{1}{2 \varepsilon}\left\|\delta_{t}^{\alpha} \omega^{k}\right\|^{2} \\
& +\frac{\varepsilon}{2} h \sum_{i=1}^{M-1}\left(c_{0}\left|2 \omega_{i}^{k-1}-\omega_{i}^{k-2}\right|+c_{1}\left|\omega_{i}^{k-n}\right|\right)^{2} \\
\leq & \frac{1}{2 \varepsilon}\left\|\delta_{t}^{\alpha} \omega^{k}\right\|^{2}+\varepsilon c_{0}^{2} h \sum_{i=1}^{M-1}\left(2 \omega_{i}^{k-1}-\omega_{i}^{k-2}\right)^{2} \\
& +\varepsilon c_{1}^{2} h \sum_{i=1}^{M-1}\left(\omega_{i}^{k-n}\right)^{2} \\
\leq & \frac{1}{2 \varepsilon}\left\|\delta_{t}^{\alpha} \omega^{k}\right\|^{2}+8 \varepsilon c_{0}^{2}\left(\left\|\omega^{k-1}\right\|^{2}+\left\|\omega^{k-2}\right\|^{2}\right) \\
& +\varepsilon c_{1}^{2}\left\|\omega^{k-n}\right\|^{2} .
\end{aligned}
$$


Substituting (43)-(45) into (42) and taking $\varepsilon=3 / 4$ in (45), we obtain

$$
\begin{aligned}
\frac{D}{4 \lambda}\left|\delta_{x} \omega^{k}\right|_{1}^{2} \leq & \frac{3 D}{2 \lambda}\left|\delta_{x} \omega^{k-n}\right|_{1}^{2} \\
& +\frac{D}{\lambda} \sum_{j=1}^{k-1}\left(d_{k-j-1}-d_{k-j}\right)\left|\delta_{x} \omega^{j}\right|_{1}^{2} \\
& +\frac{D}{\lambda} d_{k-1}\left|\delta_{x} \omega^{0}\right|_{1}^{2} \\
& +6 c_{0}^{2}\left(\left\|\omega^{k-1}\right\|^{2}+\left\|\omega^{k-2}\right\|^{2}\right) \\
& +\frac{3}{4} c_{1}^{2}\left\|\omega^{k-n}\right\|^{2} \\
\leq & \frac{D}{\lambda} \sum_{j=1}^{k-1}\left(d_{k-j-1}-d_{k-j}\right)\left|\delta_{x} \omega^{j}\right|_{1}^{2} \\
& +\frac{D}{\lambda} d_{k-1}\left|\delta_{x} \omega^{0}\right|_{1}^{2} \\
& +c_{0}^{2}\left(\left|\delta_{x} \omega^{k-1}\right|_{1}^{2}+\left|\delta_{x} \omega^{k-2}\right|_{1}^{2}\right) \\
& +\left(\frac{3 D}{2 \lambda}+\frac{1}{8} c_{1}^{2}\right)\left|\delta_{x} \omega^{k-n}\right|_{1}^{2}
\end{aligned}
$$

where Lemma 5 has been used.

Multiplying (46) by $4 \lambda / D$, we have

$$
\begin{aligned}
\left|\delta_{x} \omega^{k}\right|_{1}^{2} \leq & 4 \sum_{j=1}^{k-1}\left(d_{k-j-1}-d_{k-j}\right)\left|\delta_{x} \omega^{j}\right|_{1}^{2}+4\left|\delta_{x} \omega^{0}\right|_{1}^{2} \\
& +\frac{4 \lambda}{D} c_{0}^{2}\left(\left|\delta_{x} \omega^{k-1}\right|_{1}^{2}+\left|\delta_{x} \omega^{k-2}\right|_{1}^{2}\right) \\
& +\left(6+\frac{\lambda}{2 D} c_{1}^{2}\right)\left|\delta_{x} \omega^{k-n}\right|_{1}^{2}
\end{aligned}
$$

Denote

$$
C_{k}=\max \left\{\frac{4 \Gamma(2-\alpha)}{D} c_{0}^{2}, \frac{\Gamma(2-\alpha)}{2 D} c_{1}^{2}+6\right\}>0 .
$$

Noticing $\lambda=\tau^{\alpha} \Gamma(2-\alpha)$ for $0<\tau<1$, we have $\tau^{\alpha}<1$. Then, from (47), we obtain

$$
\begin{aligned}
\left|\delta_{x} \omega^{k}\right|_{1}^{2} \leq & 4 \sum_{j=1}^{k-1}\left(d_{k-j-1}-d_{k-j}\right)\left|\delta_{x} \omega^{j}\right|_{1}^{2}+4\left|\delta_{x} \omega^{0}\right|_{1}^{2} \\
& +C_{k}\left(\left|\delta_{x} \omega^{k-1}\right|_{1}^{2}+\left|\delta_{x} \omega^{k-2}\right|_{1}^{2}+\left|\delta_{x} \omega^{k-n}\right|_{1}^{2}\right) .
\end{aligned}
$$

From Lemmas 2 and 6, we have

$$
\begin{aligned}
& \left|\delta_{x} \omega^{k}\right|_{1}^{2} \\
& \quad \leq\left(3 C_{k}+4\right) \exp \left(3 C_{k}+4\left(1-d_{k-1}\right)\right) \max _{-n \leq j \leq 0}\left|\delta_{x} \omega^{j}\right|_{1}^{2} \\
& \quad \leq\left(3 C_{k}+4\right) \exp \left(3 C_{k}+4\right) \max _{-n \leq j \leq 0}\left|\delta_{x} \psi^{j}\right|_{1}^{2} .
\end{aligned}
$$

From Lemma 5, we have

$$
\left\|\omega^{k}\right\|_{\infty} \leq \widehat{C} \max _{-n \leq j \leq 0}\left|\psi^{j}\right|_{1}
$$

The proof is completed.

Denote $e_{i}^{k}=U_{i}^{k}-u_{i}^{k}, 0 \leq i \leq M,-n \leq k \leq N$; by subtracting (24)-(26) from (19), (22), and (23), respectively, the following error equations can be obtained:

$$
\begin{aligned}
\mathscr{A} \delta_{t}^{\alpha} e_{i}^{k} & =D\left(\delta_{x}^{2} e_{i}^{k}+\delta_{x}^{2} e_{i}^{k-n}\right)+\mathscr{A} p_{i}^{k}+R_{i}^{k}, \\
e_{i}^{k} & =0, \quad 0 \leq i \leq M,-n \leq k \leq 0, \\
e_{0}^{k} & =0 \\
e_{M}^{k} & =0,
\end{aligned}
$$

$$
1 \leq k \leq N
$$

where $p_{i}^{k}=f\left(2 U_{i}^{k-1}-U_{i}^{k-2}, U_{i}^{k-n}, x_{i}, t_{k}\right)-f\left(2 u_{i}^{k-1}-\right.$ $\left.u_{i}^{k-2}, u_{i}^{k-n}, x_{i}, t_{k}\right)$.

Similar to the proof of Theorem 9, the following convergence result can be obtained.

Theorem 10. Assume $u(x, t) \in C^{6,2}([0,1] \times(-s, T])$ is the solution of (3)-(5) and $\left\{u_{i}^{k} \mid 0 \leq i \leq M,-n \leq k \leq N\right\}$ is the solution of (24)-(26). Then, one has

$$
\left\|e^{k}\right\|_{\infty} \leq C\left(\tau^{2-\alpha}+h^{4}\right), \quad 1 \leq k \leq N
$$

where $C$ is a positive constant independent of $h$ and $\tau$.

\section{Numerical Test}

In this section, a numerical test is used to validate the performance of scheme (24)-(26). Denote the maximum error at all grid points as

$$
e(h, \tau)=\max _{1 \leq k \leq N}\left\|U^{k}-u^{k}\right\|_{\infty}
$$

the convergence order in time and space is defined, respectively, as

$$
\begin{aligned}
\text { Rate }_{\tau} & =\frac{\log \left(e\left(h, \tau_{1}\right) / e\left(h, \tau_{2}\right)\right)}{\log \left(\tau_{1} / \tau_{2}\right)}, \\
\text { Rate }_{h} & =\frac{\log \left(e\left(h_{1}, \tau\right) / e\left(h_{2}, \tau\right)\right)}{\log \left(h_{1} / h_{2}\right)} .
\end{aligned}
$$

For Rate ${ }_{\tau}$, we require $h$ to be fixed and small enough, while for Rate R $_{h} \tau$ should be fixed and small enough. 


$$
\alpha=0.2
$$

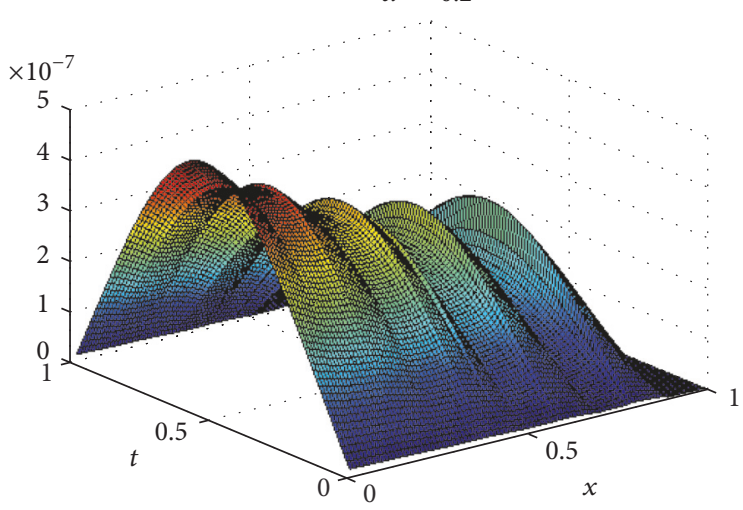

$\alpha=0.6$

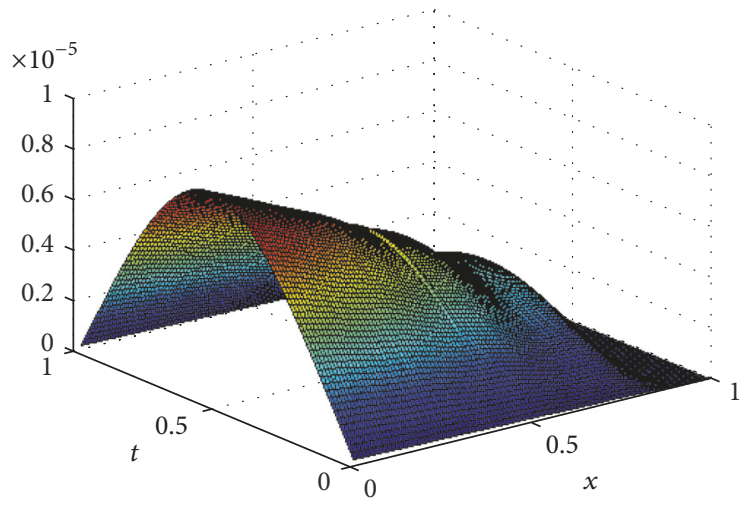

$\alpha=0.4$

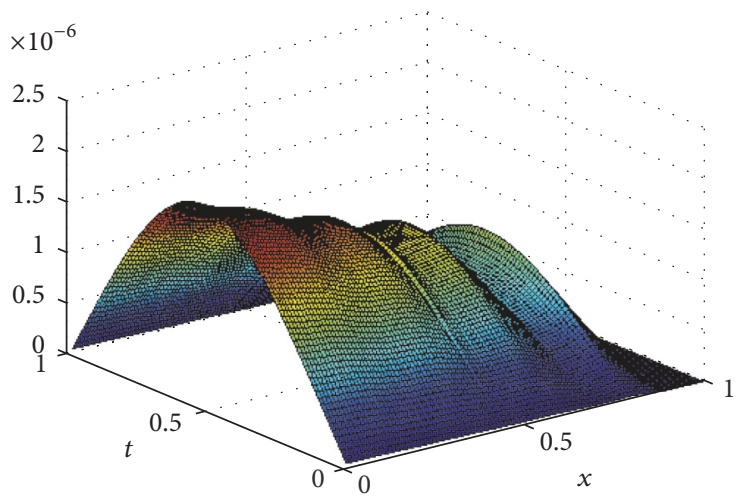

$\alpha=0.8$

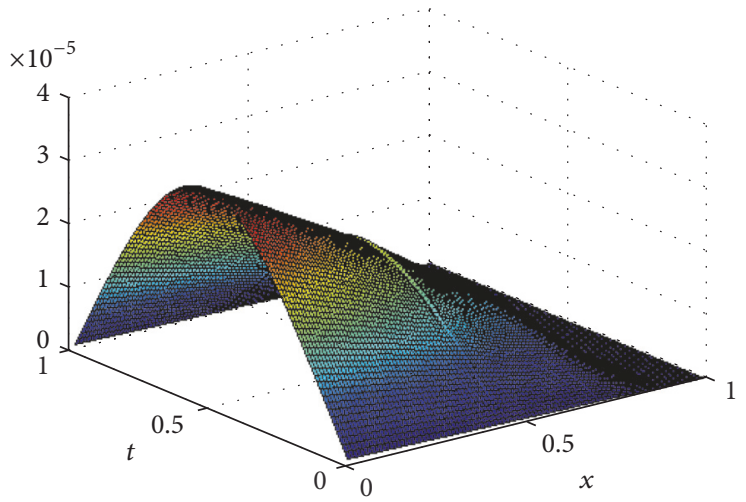

FIGURE 1: Error planes for $\tau=h=1 / 100$ for different $\alpha$ in Example 1.

Example 1. Consider the following problem:

$$
\begin{aligned}
& \frac{\partial^{\alpha} u}{\partial t^{\alpha}}=\left(\frac{\partial^{2} u(x, t)}{\partial x^{2}}+\frac{\partial^{2} u(x, t-0.1)}{\partial x^{2}}\right)-u(x, t)^{2} \\
& +u(x, t-0.1)+G(x, t), \\
& \qquad(x, t) \in(0,1) \times(0,1], \\
& \begin{aligned}
& u(x, t)= e^{x} x^{2}(1-x)^{2} t^{2+\alpha}, \\
& u(0, t)=0, x \in[0,1], t \in[-0.1,0], \\
& u(1, t)=0,
\end{aligned}
\end{aligned}
$$$$
t \in(0,1]
$$

the exact solution of (56) is $u(x, t)=e^{x} x^{2}(1-x)^{2} t^{2+\alpha}$, and

$$
\begin{aligned}
G(x, t)=e^{2 x} & x^{4}(1-x)^{4} t^{4+2 \alpha}-e^{x} x^{2}(1-x)^{2} \\
& \cdot(t-0.1)^{2+\alpha}+\frac{\Gamma(3+\alpha)}{2} t^{2} e^{x} x^{2}(1-x)^{2}
\end{aligned}
$$

$$
\begin{gathered}
-e^{x}\left(2-8 x+x^{2}+6 x^{3}+x^{4}\right) \\
\cdot\left(t^{2+\alpha}+(t-0.1)^{2+\alpha}\right)
\end{gathered}
$$

From Table 1, we can see the maximum errors between the numerical solution and the exact solution in the temporal directions for $\alpha=0.3,0.5,0.8$, respectively, where the spatial step is fixed to be $h=1 / 400$. The results show that the temporal convergence order matches well the theoretical convergence order of $2-\alpha$.

Table 2 shows the maximum errors in the spatial directions for $\alpha=0.2$ when the temporal step is fixed at $\tau=1 / 2000$. From the results, we can see that the spatial convergence order is 4 , which coincides with the theoretical result.

Figure 1 gives the error plane for $\alpha=0.2,0.4,0.6,0.8$, respectively. From this figure, we can see that the error becomes larger when a larger $\alpha$ is taken.

\section{Conclusion}

This paper presents a compact finite difference scheme for solving the fractional neutral parabolic differential equation with proportional delay. The unconditional stability and the 
TABLE 1: Maximum errors and convergence order in temporal direction with $h=1 / 400$ and $\alpha=0.3,0.5,0.8$ for Example 1 .

\begin{tabular}{|c|c|c|c|c|c|c|}
\hline \multirow{2}{*}{$\tau$} & \multicolumn{2}{|c|}{$\alpha=0.3$} & \multicolumn{2}{|c|}{$\alpha=0.5$} & \multicolumn{2}{|c|}{$\alpha=0.8$} \\
\hline & $e(h, \tau)$ & Rate $_{\tau}$ & $e(h, \tau)$ & Rate $_{\tau}$ & $e(h, \tau)$ & Rate $_{\tau}$ \\
\hline $1 / 100$ & $1.083 e-006$ & $*$ & $4.502 e-006$ & $*$ & $3.800 e-005$ & $*$ \\
\hline $1 / 200$ & $3.234 e-007$ & 1.744 & $1.571 e-006$ & 1.519 & $1.651 e-005$ & 1.203 \\
\hline $1 / 300$ & $1.598 e-007$ & 1.739 & $8.501 e-007$ & 1.515 & $1.014 e-005$ & 1.202 \\
\hline $1 / 400$ & $69.694 e-008$ & 1.737 & $5.501 e-007$ & 1.513 & $7.176 e-006$ & 1.202 \\
\hline
\end{tabular}

TABle 2: Maximum errors and convergence order in spatial direction with $\tau=1 / 2000$ and $\alpha=0.2$ for Example 1 .

\begin{tabular}{lcc}
\hline$h$ & $e(h, \tau)$ & Rate $_{h}$ \\
\hline $1 / 8$ & $7.949 e-005$ & $*$ \\
$1 / 12$ & $1.578 e-005$ & 3.988 \\
$1 / 16$ & $4.976 e-006$ & 4.011 \\
$1 / 20$ & $2.045 e-006$ & 3.985 \\
\hline
\end{tabular}

global convergence of the scheme in the maximum norm are proved. The convergence order of the considered scheme is $O\left(\tau^{2-\alpha}+h^{4}\right)$. A numerical experiment is presented to support the theoretical results and validate the efficiency of the difference scheme.

\section{Conflicts of Interest}

The authors declare that there are no conflicts of interest regarding the publication of this paper.

\section{Acknowledgments}

This research work is supported by the National Natural Science Foundation of China (11401591) and the Humanities and Social Science Foundation of the Ministry of Education of China (17YJC630236). The first author acknowledges the Project of the Scientific Research Foundation for the Returned Overseas Chinese Scholars, State Education Ministry (2013693).

\section{References}

[1] A. V. Rezounenko and J. Wu, "A non-local PDE model for population dynamics with state-selective delay: local theory and global attractors," Journal of Computational and Applied Mathematics, vol. 190, no. 1-2, pp. 99-113, 2006.

[2] B. Zubik-Kowal, "Solutions for the cell cycle in cell lines derived from human tumors," Computational and Mathematical Methods in Medicine. An Interdisciplinary Journal of Mathematical, Theoretical and Clinical Aspects of Medicine, vol. 7, no. 4, pp. 215228, 2006.

[3] D. Li, C. Zhang, and W. Wang, "Long time behavior of nonFickian delay reaction-diffusion equations," Nonlinear Analysis: Real World Applications, vol. 13, no. 3, pp. 1401-1415, 2012.

[4] Z.-X. Sun and Z. B. Zhang, "A linearized compact difference scheme for a class of nonlinear delay partial differential equations," Applied Mathematical Modelling: Simulation and
Computation for Engineering and Environmental Systems, vol. 37, no. 3, pp. 742-752, 2013.

[5] Q. Zhang and C. Zhang, "A compact difference scheme combined with extrapolation techniques for solving a class of neutral delay parabolic differential equations," Applied Mathematics Letters, vol. 26, no. 2, pp. 306-312, 2013.

[6] W. Gu, "A compact difference scheme for a class of variable coefficient quasilinear parabolic equations with delay," Abstract and Applied Analysis, Article ID 810352, Art. ID 810352, 8 pages, 2014.

[7] D. Li, C. Zhang, and J. Wen, "A note on compact finite difference method for reaction-diffusion equations with delay," Applied Mathematical Modelling: Simulation and Computation for Engineering and Environmental Systems, vol. 39, no. 5-6, pp. 1749-1754, 2015.

[8] C. R. Jin, Z. H. Yu, and R. N. Qu, "An implicit difference scheme for solving a neutral delay parabolic differential equation," Journal of Shandong University. Natural Science. Shandong Daxue Xuebao. Lixue Ban, vol. 46, no. 8, pp. 13-16, 2011.

[9] R. Metzler and J. Klafter, "The random walk's guide to anomalous diffusion: a fractional dynamics approach," Physics Reports, vol. 339, pp. 1-77, 2000.

[10] S. Chen, F. Liu, I. Turner, and V. Anh, "An implicit numerical method for the two-dimensional fractional percolation equation," Applied Mathematics and Computation, vol. 219, no. 9, pp. 4322-4331, 2013.

[11] F. A. Rihan, "Computational methods for delay parabolic and time-fractional partial differential equations," Numerical Methods for Partial Differential Equations, vol. 26, no. 6, pp. 1556-1571, 2010.

[12] Q. Zhang, M. Ran, and D. Xu, "Analysis of the compact difference scheme for the semilinear fractional partial differential equation with time delay," Applicable Analysis: An International Journal, vol. 96, no. 11, pp. 1867-1884, 2017.

[13] Z. Sun and X. Wu, "A fully discrete difference scheme for a diffusion-wave system," Appl. Numer. Math, vol. 56, pp. 193-209, 2006.

[14] S. Chen, F. Liu, P. Zhuang, and V. Anh, "Finite difference approximations for the fractional Fokker-Planck equation," Applied Mathematical Modelling: Simulation and Computation for Engineering and Environmental Systems, vol. 33, no. 1, pp. 256-273, 2009.

[15] Z. Z. Sun, The numerical methods for partial equations, Science Press, Beijing, China, 2005. 


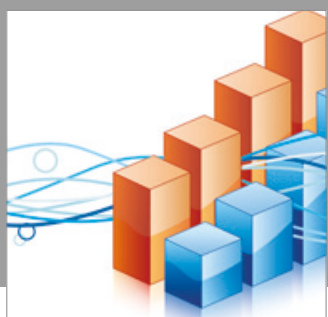

Advances in

Operations Research

vatersals

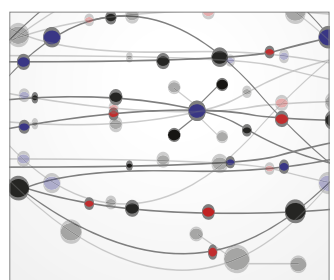

\section{The Scientific} World Journal
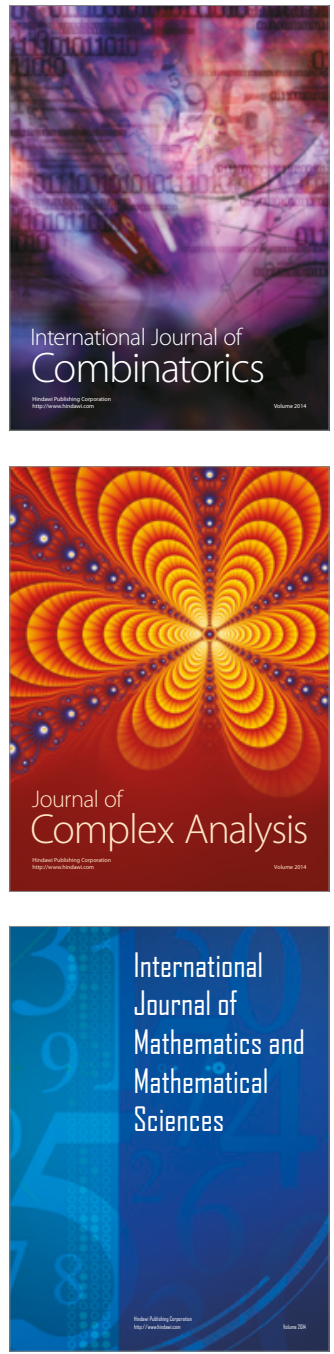
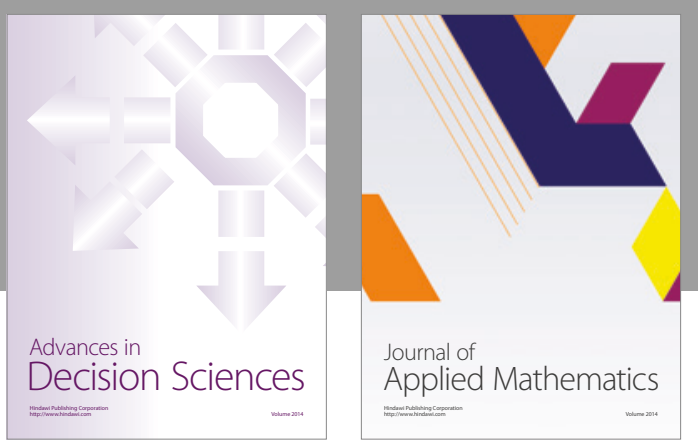

Algebra

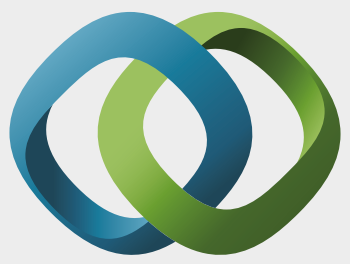

\section{Hindawi}

Submit your manuscripts at

https://www.hindawi.com
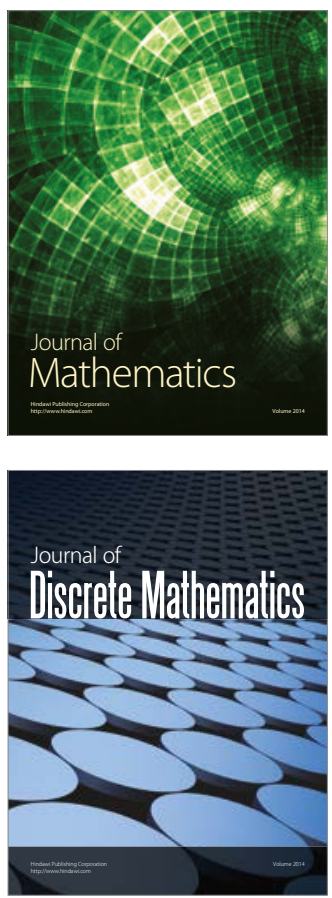

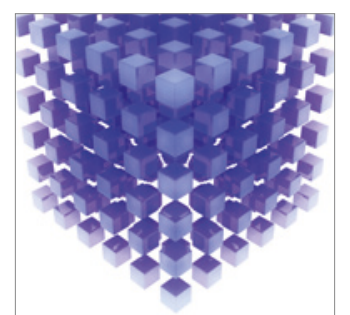

Mathematical Problems in Engineering
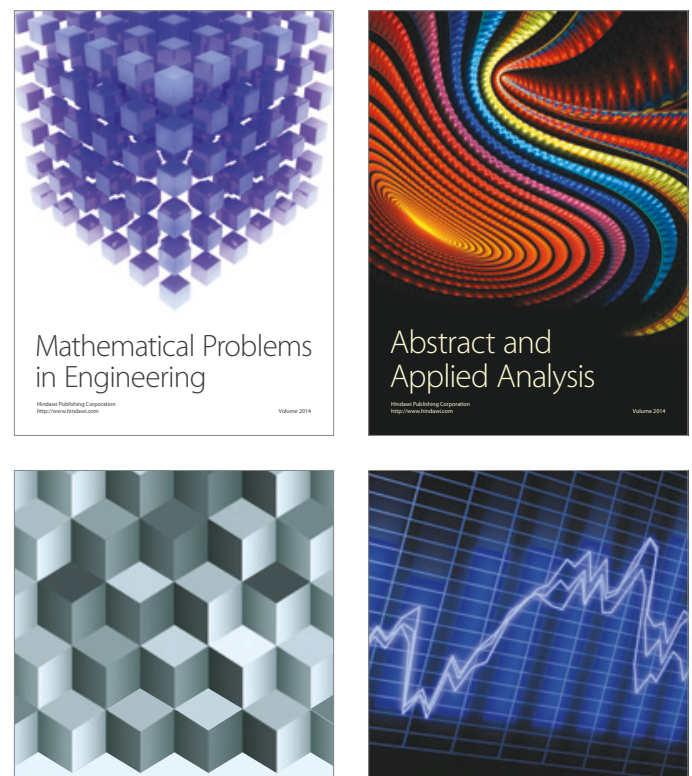

Journal of

Function Spaces

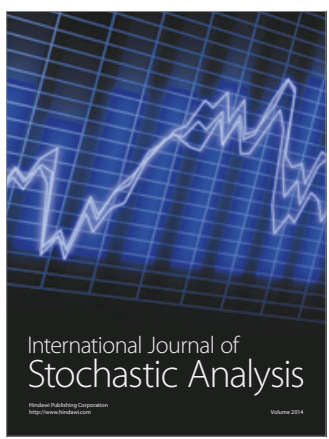

Probability and Statistics
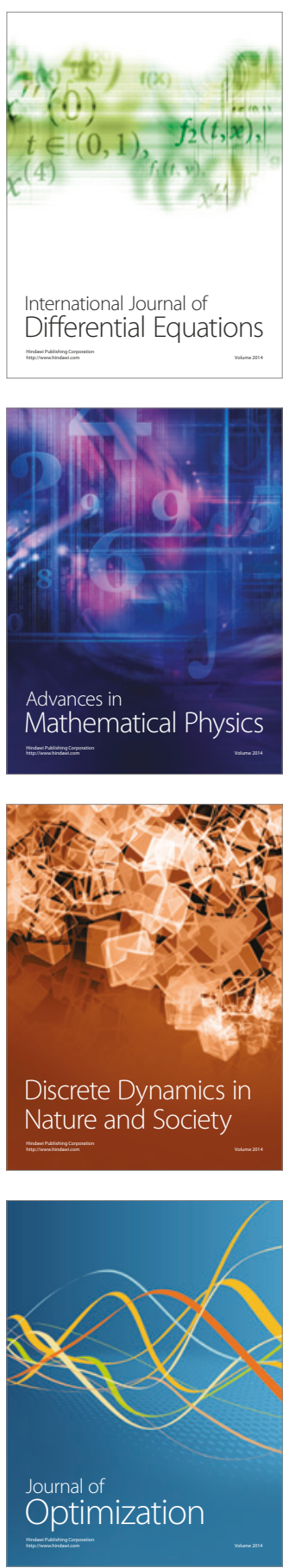